Original paper

\title{
Characterization of passive dosimeters in proton pencil beam scanning - A EURADOS intercomparison for mailed dosimetry audits in proton therapy centres
}

\author{
M. De Saint-Hubert ${ }^{a, *}$, C. De Angelis ${ }^{b}$, Ž. Kneževićc ${ }^{c}$, B. Michalec ${ }^{\text {d }}$, B. Reniers ${ }^{\mathrm{e}}$, E. Pyszka ${ }^{\mathrm{d}}$, \\ L. Stolarczyk $^{\mathrm{d}}$, J. Swakon ${ }^{\mathrm{d}}$, G. Foltynska ${ }^{\mathrm{d}}$, A. Wochnik ${ }^{\mathrm{d}}$, A. Parisi ${ }^{\mathrm{a}}$, M. Majer ${ }^{\mathrm{c}}$, R.M. Harrison ${ }^{\mathrm{f}}$, \\ R. Kopec ${ }^{\mathrm{d}}$, F. Vanhavere ${ }^{\mathrm{a}}$, P. Olko ${ }^{\mathrm{d}}$

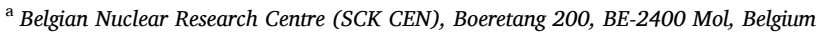 \\ b Istituto Superiore di Sanità (ISS), Viale Regina Elena 299, 00161 Rome, Italy \\ ${ }^{\mathrm{c}}$ Ruđer Bošković Institute (RBI), Bijenička 54, Zagreb, Croatia \\ d Institute of Nuclear Physics Polish Academy of Sciences (IFJ PAN), Radzikowskiego 152, 31-342 Krakow, Poland \\ ${ }^{\mathrm{e}}$ Research Group NuTeC, University Hasselt (UH), Agoralaan Gebouw H, B-3590 Diepenbeek, Belgium \\ ${ }^{\mathrm{f}}$ University of Newcastle, Newcastle Upon Tyne NE2 4HH, UK
}

\section{A R T I C L E I N F O}

\section{Keywords:}

Alanine

Luminescence dosimetry

Mail auditing

Proton therapy

\begin{abstract}
A B S T R A C T
The lack of mailed dosimetry audits of proton therapy centres in Europe has encouraged researchers of EURADOS Working Group 9 (WG9) to compare response of several existing passive detector systems in therapeutic pencil beam scanning.

Alanine Electron Paramagnetic Resonance dosimetry systems from 3 different institutes (ISS, Italy; UH, Belgium and IFJ PAN, Poland), ${ }^{\text {nat }} \mathrm{LiF}: \mathrm{Mg}$, Ti (MTS-N) and ${ }^{\text {nat }} \mathrm{LiF}: \mathrm{Mg}, \mathrm{Cu}, \mathrm{P}(\mathrm{MCP}-\mathrm{N})$ thermoluminescent dosimeters (TLDs), GD-352M radiophotoluminescent glass dosimeters (RPLGDs) and $\mathrm{Al}_{2} \mathrm{O}_{3}: \mathrm{C}$ optically stimulated dosimeters (OSLDs) were evaluate. Dosimeter repeatability, batch reproducibility and response in therapeutic Pencil Beam Scanning were verified for implementation as mail auditing system.

Alanine detectors demonstrated the lowest linear energy transfer (LET) dependence with an agreement between measured and treatment planning system (TPS) dose below $1 \%$. The OSLDs measured on average a $6.3 \%$ lower dose compared to TPS calculation, with no significant difference between varying modulations and ranges. Both GD-352M and MCP-N measured a lower dose than the TPS and luminescent response was dependent on the LET of the therapeutic proton beam. Thermoluminescent response of MTS-N was also found to be dependent on the LET and a higher dose than TPS was measured with the most pronounced increase of $11 \%$.

As alanine detectors are characterized by the lowest energy dependence for different parameters of therapeutic pencil beam scanning they are suitable candidates for mail auditing in proton therapy. The response of luminescence detector systems have shown promises even though more careful calibration and corrections are needed for its implementation as part of a mailed dosimetry audit system.
\end{abstract}

\section{Introduction}

Every radiotherapy unit used for regular patient treatment has to undergo dosimetry audits to ensure an accurate dose delivery to the patient and to assist radiotherapy centres in maintaining the performance of their dosimetry systems within acceptable limits. Independent external dosimetric audits are a necessary part of a comprehensive quality assurance programme in radiation oncology. Since 1969, the
International Atomic Energy Agency (IAEA) together with the World Health Organization (WHO) has been performing audits by mail in radiotherapy centres across the world [1]. Also IAEA has established an international network of national Secondary Standard Dosimetry Laboratories (SSDL) in order to promote accuracy, traceability and cooperation within member states and among others provides dosimetry audit services [2]. The UK National Physics Laboratory offers a wide range of radiotherapy dosimetry audits and operates a mailed alanine

\footnotetext{
* Corresponding author.

E-mail address: mdsainth@sckcen.be (M. De Saint-Hubert).
} 
reference dosimetry service. The European Quality Assurance Laboratory at Villejuif-Paris, under the auspices of European Society for Radiotherapy and Oncology (ESTRO), has audited photon and electron beams since 1998 [3]. Moreover, several national programmes for mail auditing of radiotherapy centres in Europe have been widely implemented [3-6]. In the USA, since 2008, the Imaging and Radiation Oncology Core (IROC-C), performs audits of proton therapy units [7].

At present, dosimetry auditing is still not available in Europe for proton therapy centers. Nonetheless, the continuous exploration of the benefits of proton therapy is inspiring a growing and large scale construction of new proton therapy centres across Europe. In all new proton therapy facilities Pencil Beam Scanning (PBS) technology with isocentric gantries is applied, a technology which has been commercially available from the beginning of this decade. Auditing programs would contribute to dosimetry harmonisation among proton therapy centres, which is currently challenged by the lack of international and national primary dose standards for proton beams. In addition, there are no international recommendations for the procedures of reference dosimetry as it is the case for Double Scattering beams, as explained in TRS-398 [8]. All these factors encouraged the researchers of EURADOS Working Group 9 to test, with proton beams, widely available dosimetry systems used extensively for auditing conventional radiotherapy units. In the latter, thermoluminescent detectors (TLD), radiophotoluminescence (RPL) or Electron Paramagnetic Resonance (EPR) alanine detectors are sent by specialized dosimetry laboratories to the radiotherapy clinics and are analysed upon return [9]. Although auditing programs are predominantly based on TLDs $[1,10]$, alanine is considered to be more attractive in comparison to TLDs for several reasons including intrinsic tissue equivalency, weak energy dependence, dose rate independence and low fading [11]. In the past, some national audit programs have used alanine and/or TLD powder such as in Italy [4], Belgium [5], Poland or Czech Republic [12,13]. While the successful introduction and development of carbon doped aluminium oxide $\left(\mathrm{Al}_{2} \mathrm{O}_{3}: \mathrm{C}\right)$ for optically stimulated luminescence detectors (OSLDs) has led to its routine use as a passive personal dosimeter, its use in radiotherapy dosimetry is still not broadly applied [14]. In the USA, the IROC$\mathrm{C}$ audits of proton therapy units use TLDs and $\mathrm{Al}_{2} \mathrm{O}_{3}: \mathrm{C}$ detectors based on OSL [7]. Major advantages can be fast readout and options of 2D OSL dose imaging [15]. On the other hand, commercially available radiophotoluminescence glass dosimeters (RPL-GD type) have been increasingly used in dosimetry in conventional radiation therapy $[16,17]$ and have also been used for photon dosimetry audits [9]. Low energy dependence, low fading effects, excellent dose linearity and readout repeatability make these RPL-GD types promising for auditing $\theta f$ radiotherapy centres $[9,18,19]$.

In mailed dosimetric audits performed at IAEA in last 20 years, the stated and the measured doses should not differ by more than $5 \%$ [20]. To achieve this level of precision, the uncertainties associated with dependency of dose, energy, ionization density (LET), reproducibility, repeatability, batch uniformity, etc. must be much lower. Therefore the current study evaluates the response of a number of dosimetry systems such as MCP-N (LiF:Mg, Cu, P TLD), MTS-N (LiF:Mg, Ti TLD), GD-352M (RPLGD), $\mathrm{Al}_{2} \mathrm{O}_{3}$ : $\mathrm{C}$ (OSLD) and alanine detectors in the same controlled conditions in a clinical proton therapy centre (Cyclotron Centre Bronowice, IFJ PAN, Kraków, Poland). In particular, the response of alanine detectors from three different institutes with different EPR reader systems and their specific protocols was tested using in the same PBS technique. Moreover, two of these participating centers perform national audits of conventional radiotherapy centers in their countries (Italy and Belgium) providing important and valid experience towards future auditing programs in PT. Within this framework, this study focused on the response of the detectors in different proton fields (beam modulation and range) as well as testing their repeatability, reproducibility and batch reproducibility.

\section{Materials and methods}

In this section, the dosimeters and dosimetry systems are described by specifying each detector type, system and specific protocols used by the different institutes followed by a section of dosimeter calibration. Thereafter, we describe the tests performed for comparison between the detectors including dosimeter repeatability, reproducibility and batch reproducibility, as well as the response in Co- 60 reference beams, according to TRS-398. Finally, we describe the irradiations performed in pencil beam scanning for 9 different SOBP configurations including a Monte Carlo simulation of the LET at the detector's position.

\subsection{Dosimeters and dosimetry systems}

\subsubsection{Electron paramagnetic resonance}

2.1.1.1. Bruker ELEXSYS spectrometer. The Istituto Superiore di Sanità (ISS) alanine dosimeters were supplied by Gamma-Service (Leipzig, Germany). They are cylindrically shaped with a diameter of $4.80 \pm 0.04$ $\mathrm{mm}$, height of $2.98 \pm 0.04 \mathrm{~mm}$ and mass of $65.0 \pm 0.5 \mathrm{mg}$. Their composition is $96 \%$ and $4 \%$ by weight of alanine and ligand material, respectively. EPR measurements were performed with a Bruker (Karlsruhe, Germany) ELEXSYS spectrometer operating in the X-band and equipped with a Bruker SHQ cavity, in controlled environmental conditions. The EPR spectra were acquired using the following parameters: $2 \mathrm{~mW}$ microwave power, $8 \mathrm{G}$ modulation amplitude, $20 \mathrm{~s}$ sweep time, 25 $\mathrm{G}$ sweep field. Each measurement was carried out using a stack of three alanine pellets. Each stack was measured three times, each time varying the position of the pellets in the stack. The mean value of the three repeated measurements was then correlated to dose in water. A more detailed description of the protocol is reported elsewhere [21]. The alanine dosimeters were calibrated in terms of absorbed dose-to-water $\left(D_{\mathrm{w}}\right)$ in a Co-60 source at the Italian Primary Standard Dosimetry Laboratory (PSDL) (ENEA-INMRI) in the $5-15$ Gy range. To take into account the variations in the EPR signal due to different irradiation temperatures, the correction factor provided by the manufacturer was applied. Uncertainty in the alanine dose was evaluated as prediction interval [22]. This method provides an estimate of the interval in which a future observation will fall with a given probability (in our case 68\%) on the basis of the calibration design (i.e. number of calibration doses, specific dose values, number of dosimeters used for each dose and number of dosimeters used as unknown samples irradiated in the same experimental conditions) and parameters of the calibration curve (i.e. slope and standard error). The uncertainty evaluated following this approach was $1 \%$ at $10 \mathrm{~Gy}$.

2.1.1.2. Bruker EMXmicro spectrometer. The UHasselt (UH) alanine pellets (Harwell, Oxfordshire, UK) have a cylindrical shape $(\varnothing=4.8 \pm$ $0.1 \mathrm{~mm} ; \mathrm{h}=2.70 \pm 0.01 \mathrm{~mm}$ ) and mass $\mathrm{m}=59.8 \mathrm{mg}$ with composition 90.9 and $9.1 \%$ by weight of $\mathrm{L}-\alpha$-alanine and paraffin, respectively. The detectors were read out in a Bruker EMXmicro spectrometer $(900$ magnet, X-band) equipped with a high sensitivity resonator ER4119HSW1. The spectrometer settings, environmental conditions and applied corrections have been described elsewhere [5,23]. A total of 5 separate spectra were acquired for each detector after subsequent equal rotation alanine pellets.

The dose-to-water was determined from the average reading of 6 detectors in terms of dose-normalized amplitudes $\left(A_{D}\right)$ - formula (1) in reference [5] - and applying corrections for temperature $\left(k_{\mathrm{T}}\right)$ and fading $\left(k_{\mathrm{f}}\right)$. A set of dosimeters irradiated in a Co-60 reference beam in water $\left(D_{w}\right)$ between 2 and 25 Gy against the primary water calorimetry standard at PTB was used for calibration [5].

2.1.1.3. Bruker ESP 300 spectrometer. The Institute of Nuclear Physics Polish Academy of Sciences (IFJ PAN) uses Gamma Service pellet- 


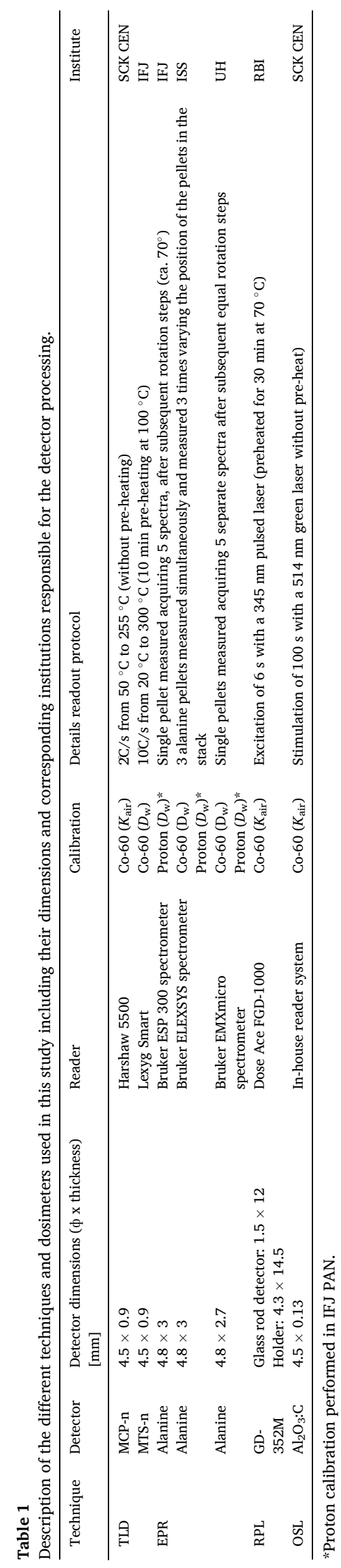

shaped alanine dosimeters ( $\phi=4.80 \pm 0.04 \mathrm{~mm}, h=2.98 \pm 0.04 \mathrm{~mm}, M$ $=65.0 \pm 0.5 \mathrm{mg}$ ). EPR measurements were performed with a Bruker ESP 300 spectrometer operating in the X-band and equipped with a Bruker TM 110 cylindrical cavity. The EPR spectra were measured using the following parameters: $8 \mathrm{~mW}$ microwave power, $10 \mathrm{G}$ modulation amplitude, $20 \mathrm{~s}$ sweep time, $25 \mathrm{G}$ sweep field. Each measurement was performed using one alanine pellet placed on a quartz spacer to ensure its reproducible position in the center of the cavity. Each pellet was measured 5 times, rotated ca. $70^{\circ}$ after each measurement to average the signal. The mean value of the 5 repeated measurements was then correlated to dose to water. No reference Co- 60 calibration was performed for IFJ PAN as alanine dosimeters are standardly calibrated in a $60 \mathrm{MeV}$ beam produced in AIC-144 cyclotron at IFJ PAN.

2.1.1.4. Radiophotoluminescence dosimetry. The Ruđer Bošković Institute (RBI) used a Dose Ace system consisting of radiophotoluminescent (RPL) glass dosimeters type GD-352M (GD-300 series) and FDG-1000 reader(manufacturer AGC Techno Glass, Japan) [24]. The GD-352M dosimeterconsists of a silver activated phosphate glass rod inserted into a plastic holder (see table 1). In comparison to RPL-GD type GD-302 $\mathrm{M}$, used in other studies, GD-352M has an energy compensation filter. The readout was performed using a Dose Ace FGD-1000 automatic reader equipped with a pulsed ultraviolet (UV) laser used for the readout of the dosimeters. Prior to irradiation, the dosimeters were annealed for $1 \mathrm{~h}$ at $400{ }^{\circ} \mathrm{C}$ and after irradiation, and before readout, the dosimeters were preheated for $20 \mathrm{~min}$ at $70^{\circ} \mathrm{C}$ to stabilize the luminescence centers. As the readout process will not eliminate luminescence centres created by irradiation and light is emitted every time the laser pulses excite the glass, RPLGDs can be read out multiple times. Each RPL-GD readout was repeated at least 5 times and the mean readout dose was calculated. RPLGDs were calibrated in terms of kerma free in air $\left(K_{\text {air }}\right)$, using a Co-60 source $\left(K_{\text {air }}=1 \mathrm{~Gy}\right)$ in the Secondary Standard Dosimetry Laboratory (SSDL) at RBI [25]. Conversion to $D_{\mathrm{w}}$ was based on experimentally determined factors $\left(D_{w} / K_{\text {air }}=1.12\right)$ which were in good agreement with the ratio of mass energy absorption coefficients for water to air for Co-60 $[26,27]$. Individual factors (IF) for RPL-GD dosimeters were not applied. The detailed procedures of calibration, dose calculations, characteristics and principles of RPL-GDs are described in a previously published paper [28].

\subsection{Thermoluminescence dosimetry}

\subsection{1. $M C P-n$}

MCP-N (LiF:Mg, Cu, P) are high sensitivity thermoluminescence detectors produced by Radcard (former TLD Poland), Krakow, Poland. These small cylindrical pellets have a $4.5 \mathrm{~mm}$ diameter and $0.9 \mathrm{~mm}$ thickness. Annealing was performed always $24 \mathrm{~h}$ before the irradiation by heating the pellets at $240{ }^{\circ} \mathrm{C}$ for 12 min then cooling down in a $-10{ }^{\circ} \mathrm{C}$ freezer for $5 \mathrm{~min}$. The Belgian nuclear research centre (SCK CEN) performed the readout in a Harshaw 5500 reader using a constant heating rate of $2 \mathrm{C} / \mathrm{s}$ from $50{ }^{\circ} \mathrm{C}$ to $255{ }^{\circ} \mathrm{C}$ (without pre-heating). Glow curves were processed and signal intensity was quantified by integrating the counts of the main peak $\left(190-255^{\circ} \mathrm{C}\right)$. Due to batch inhomogeneity, each MCP-N detector was corrected for its specific sensitivity by calculating individual factors (IF), i.e. ratio of specific detector intensity to an average intensity of the reference group of detectors as measured in Co60 gamma rays. The calibration in terms of kerma free in air $\left(K_{\text {air }}\right)$ was performed by irradiating calibration detectors $(n=6)$, to an absorbed dose of $2 \mathrm{~Gy}$, in a Co-60 radiation beam in electronic equilibrium condition in the Secondary Standard Dosimetry Laboratory (SSDL) of the Belgian Nuclear research centre. Measured doses were calculated as described previously [28] and expressed as absorbed dose-to-water $\left(D_{w}\right)$ using the conversion factor $D_{w} / K_{\text {air }}=1.11$ determined by the ratio of mass energy absorption coefficient for water to air for the energy of Co60 [27]. 


\subsection{2. $M T S-n$}

MTS-N (LiF:Mg,Ti) are thermoluminescent detectors developed at IFJ PAN. Similar to MCP-N they are produced in the form of pellets 4.5 $\mathrm{mm}$ in diameter and $0.9 \mathrm{~mm}$ thick. Annealing was performed by heating the pellets at $400{ }^{\circ} \mathrm{C}$ for $1 \mathrm{~h}$ and $100{ }^{\circ} \mathrm{C}$ for $2 \mathrm{~h}$. After the irradiation, detectors were pre-heated before readout at $100 \mathrm{C}$ for $10 \mathrm{~min}$. IFJ PAN performed the readout in a Lexyg Smart reader (Freiberg Instruments $\mathrm{GmbH}$ ) using a heating rate of $10^{\circ} \mathrm{C} / \mathrm{s}$ from $20^{\circ} \mathrm{C}$ to $300^{\circ} \mathrm{C}$. Glow curves were processed and signal intensity was quantified by integrating the counts of the main peak $\left(175-250^{\circ} \mathrm{C}\right)$. In the same way as for MCP-N, IF, were applied for batch inhomogeneity. The calibration in terms of absorbed dose-to-water $\left(D_{\mathrm{w}}\right)$ was performed by irradiating calibration detectors with 1, 2 and 3 Gy in a Co-60 beam (TRS398, 2000).

\subsection{Optically stimulated luminescence dosimetry}

Even though OSLDs have not yet been used in audit programs, we considered them in the study due to their potentials for $2 \mathrm{D}$ imaging for future auditing programs. The response of thin OSLDs cylinders made of carbon doped aluminum oxide $\left(\mathrm{Al}_{2} \mathrm{O}_{3}: \mathrm{C}[29]\right)$ produced by Landauer Inc., USA, was characterized in this work. Before each irradiation, the detectors were bleached by exposing them to daylight for one day. The readout was performed using a reader system developed in-house and based on a Melles Griot 35 LAP 431 argon-ion laser [30]. The detectors were stimulated for $100 \mathrm{~s}$ with a $514 \mathrm{~nm}$ green light.

The signal was quantified as the integral of all collected light (area under the curve). The calibration in terms of kerma free in air $\left(K_{\text {air }}\right)$ was performed by irradiating calibration detectors $(n=6)$, with $2 \mathrm{~Gy}$, in a Co-60 radiation beam in electronic equilibrium condition in the Secondary Standard Dosimetry Laboratory (SSDL) of the Belgian Nuclear research centre. Measured doses were calculated as described previously [28] and expressed as absorbed dose-to-water $\left(D_{w}\right)$ using the conversion factor $D_{w} / K_{\text {air }}=1.11$ determined by the ratio of mass energy absorption coefficient for water to air for the energy of Co-60 [27].

\subsection{Dosimeter calibrations}

As mentioned in Table 1 detectors were calibrated in Co-60 and/or in proton beams. Details about Co-60 calibrations are reported in the dosimetry sections describing the specific protocols used in the different institutes. The proton calibration, as performed in IFJ PAN is described below.

\subsection{Proton calibration}

In order to compare alanine results from different Institutes, for ISS and $\mathrm{UH}$ alanine detectors conversion coefficients were applied to convert the result of their Co-60 calibrations to proton dose. For both Institutes, the conversion factors were determined by irradiating a set of 9 alanine detectors on the therapeutic eye beam line at the Proteus C235 cyclotron at IFJ PAN with a parallel, collimated, horizontal proton beam with an initial nominal energy $60 \mathrm{MeV}$. Dosimeters were placed in a PMMA holder and placed in the centre of SOBP $\left(\operatorname{LET}_{\mathrm{d}}=2.93 \mathrm{keV} / \mu \mathrm{m}\right.$, modulation for the entire range of $30 \mathrm{~mm}$ ) produced with the rotating PMMA energy modulator. For each Institute, the conversion factors were evaluated as the mean value of the ratio of the proton dose to dose calculated applying the institutes' Co-60 calibration. The conversion coefficients from Co-60 to proton calibrated beams were 1.013 and 1.033 for ISS and UH, respectively.

Calibration of alanine detectors from IFJ PAN was performed at the therapeutic eye-line at the AIC-144 cyclotron with protons of nominal energy of $60 \mathrm{MeV}$. This beam line, used for patient treatment between 2011 and 2016, allows for identical calibration conditions as the C-235. Both beam lines have metrological traceability to the Secondary Standard Dosimetry Laboratory at the National Institute of Oncology in Warsaw by Co- 60 calibrated ionization chambers.

\section{Dosimeter repeatability, reproducibility and batch reproducibility}

As mail dosimetry audits require a high level of precision the uncertainty associate to dosimeter reproducibility and repeatability and batch uniformity must be as low as possible. In this section we describe the tests performed to estimate these uncertainties and allow comparison between detector systems.

Dosimeter repeatability was evaluated for dosimetry systems with nondestructive readout, such as alanine and RPL-GD. The readout is performed at least three times according to the specific protocols of the participating institutes (see detector systems). The coefficient of variation of these repeated protocol readings was calculated for 10 detectors and the average coefficient of variation represents the dosimeter repeatability according to the institute's protocol.

Dosimeter reproducibility was evaluated for TLDs, OSLD and RPL-GDs by analyzing the results of 5 repeated irradiations and consequent readout cycles $(c=5)$ with Co-60 gamma rays. This was not done for alanine detectors because the signal cannot be erased from the detector. The coefficient of variation on the average signal is measured for 10 detectors and averaged to obtain the dosimeter reproducibility.

Batch reproducibility was evaluated as the average standard deviation of the different dosimeters from the same batch when irradiated in Co60 gamma rays (1 Gy for TLD, OSLD and RPL-GD and $10 \mathrm{~Gy}$ for alanine). This was done for all dosimetry systems by calculating the coefficient of variation on the average of 10 detector readings according to the protocols used in the institutes.

\section{Reference irradiation in Co-60 (TRS-398)}

Alanine pellets were irradiated at the Italian Primary Standard Dosimetry Laboratory (PSDL in ENEA-INMRI, Italy) with a Co-60 gamma ray source, following the TRS-398 IAEA protocol $(\mathrm{SSD}=100$ $\mathrm{cm}, 10 \mathrm{~cm} \times 10 \mathrm{~cm}$ field size) [8]. Four different irradiations with $10 \mathrm{~Gy}$ were performed under the same experimental conditions. In each irradiation dosimeters from different institutes were simultaneously positioned in the holder.

\section{Pencil beam scanning irradiations}

Detectors were positioned in a standard $30 \mathrm{~cm} \times 30 \mathrm{~cm} \times 30 \mathrm{~cm}$ PTW water phantom 41023 dedicated to horizontal beam irradiation. Comparable holders were used with slightly different inserts able to host up to 6 TLDs, 6 OSLDs, 6 alanine detectors or 3 RPL-GDs (see Fig. 1).

The measuring depth was adjusted by means of a caliper on the phantom top and adjusted to fit the reference position of each detector (i.e. the centre of the sensitive volume of the detector) to the center of the SOBP in the different configurations.

All proton beam irradiations were performed using the dedicated IBA scanning gantry at the Cyclotron Centre Bronowice (Kraków) using the Proteus C-235 therapy system. The SOBP proton range (from range $5 \mathrm{~cm}$ to range $25 \mathrm{~cm}$ ) as well as modulation (from modulation $5 \mathrm{~cm}$ to modulation $20 \mathrm{~cm}$ ) width were gradually modified to determine the dosimeter response in 9 different SOBP configurations (see table 2). In table 2 an overview is given with corresponding definition of the abbreviation used throughout the text. For full range modulations (R5M5 and R20M20), the beam energy was reduced by the range shifter mounted on the scanning gantry. A $10 \mathrm{~cm} \times 10 \mathrm{~cm}$ field size was always used. All plans for the phantom measurements were prepared using an Eclipse 13.6 Treatment Planning System.

\subsection{Monte Carlo simulation}

To achieve a high level of precision for mail auditing in PT, the dependency of response to the ionization density (LET) should be as low as possible. In order to understand the response of the different detectors in 


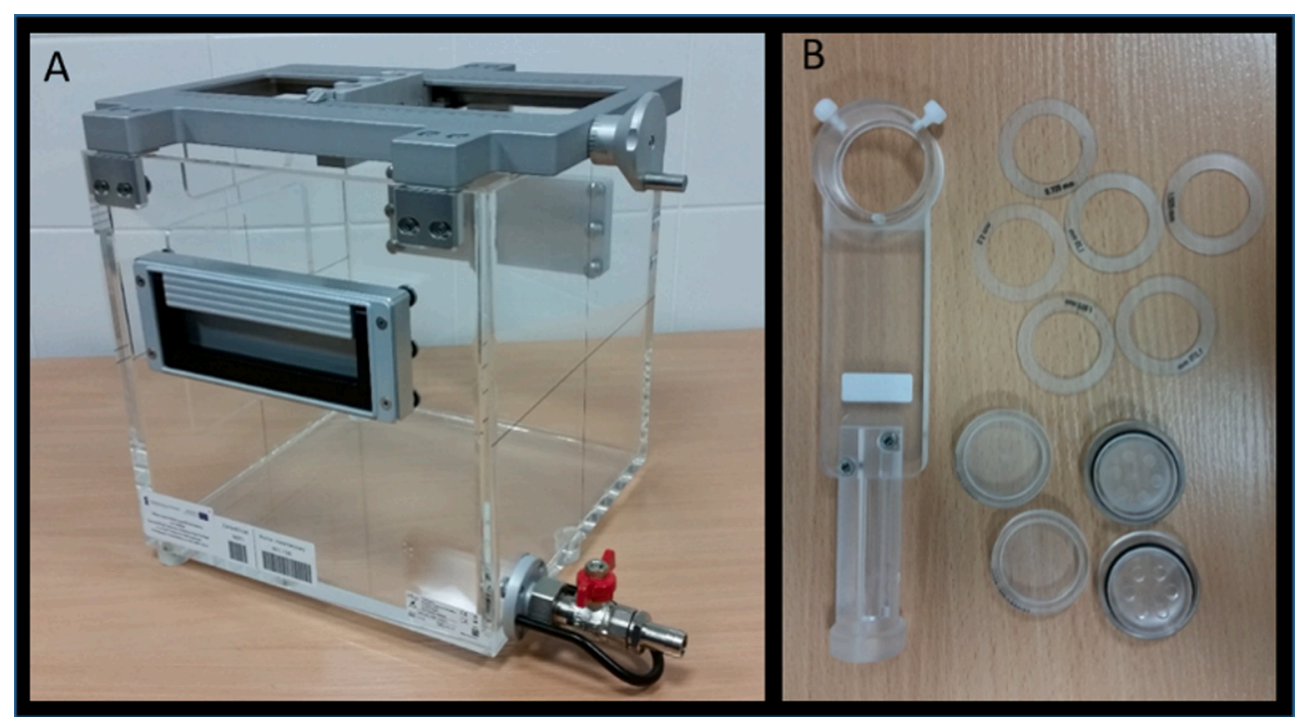

Fig. 1. PTW 41023 phantom (A) with two examples of TLD and alanine adapters (B).

Table 2

$\mathrm{LET}_{\mathrm{f}}$ and $\mathrm{LET}_{\mathrm{d}}$ calculated for different modulations (5-20 cm) and ranges (5-25 $\mathrm{cm}$ ) of proton beam scanning using MCNPx Monte Carlo code.

\begin{tabular}{|c|c|c|c|c|c|}
\hline & Range & Modulation & Definition & $\begin{array}{l}\text { MCNPx } \\
\mathrm{LET}_{\mathrm{f}} \\
(\mathrm{keV} / \mu \mathrm{m})\end{array}$ & $\begin{array}{l}\text { MCNPx } \\
\mathrm{LET}_{\mathrm{d}} \\
(\mathrm{keV} / \mu \mathrm{m})\end{array}$ \\
\hline \multirow{4}{*}{$\begin{array}{l}\text { Different } \\
\text { modulation }\end{array}$} & $20 \mathrm{~cm}$ & $5 \mathrm{~cm}$ & R20M5 & 1.55 & 2.40 \\
\hline & $20 \mathrm{~cm}$ & $10 \mathrm{~cm}$ & R20M10 & 1.20 & 1.84 \\
\hline & $20 \mathrm{~cm}$ & $15 \mathrm{~cm}$ & R20M15 & 1.01 & 1.59 \\
\hline & $20 \mathrm{~cm}$ & $20 \mathrm{~cm}$ & R20M20 & 0.89 & 1.44 \\
\hline \multirow[t]{5}{*}{ Different range } & $5 \mathrm{~cm}$ & $5 \mathrm{~cm}$ & R5M5 & 1.62 & 2.49 \\
\hline & $10 \mathrm{~cm}$ & $5 \mathrm{~cm}$ & R10M5 & 1.60 & 2.47 \\
\hline & $15 \mathrm{~cm}$ & $5 \mathrm{~cm}$ & R15M5 & 1.58 & 2.45 \\
\hline & $20 \mathrm{~cm}$ & $5 \mathrm{~cm}$ & R20M5 & 1.55 & 2.40 \\
\hline & $25 \mathrm{~cm}$ & $5 \mathrm{~cm}$ & R25M5 & 1.54 & 2.41 \\
\hline
\end{tabular}

these configurations and as function of varying LET we simulated the LET fluence in water at the location of the detector using the Monte Carlo code MCNPx 2.7.0 [31]. We used the tally type 4 (F4) to simulate proton energy flux in combination with the LET special tally treatment, FTn LET, that can be applied to track length tallies to record flux as a function of stopping power instead of energy [31]. We used logarithmic binning corresponding to 100 bins between $0.1 \mathrm{keV} / \mu \mathrm{m}$ and $100 \mathrm{keV} /$ $\mu \mathrm{m}$. The geometry of the water phantom used in the experiment was modelled in MCNPx as well as the physical range shifter used for the configuration with full range modulations (R5M5 and R20M20). The range shifter of $41.96 \mathrm{~mm}$ water equivalent thickness was placed at 2.46 $\mathrm{cm}$ from the nozzle and isocenter range shifter distance was $36.85 \mathrm{~cm}$. The beam size was $10 \times 10 \mathrm{~cm}^{2}$ and source input was derived from the Treatment Planning System (TPS) by defining the proton energies (layers) with corresponding weightings and beam size. A total number of $10^{8}$ proton particles were transported for the calculation with a resulting statistical uncertainty below $0.1 \%$.

For all configurations we calculated the LET probability distribution from the unrestricted proton LET calculations (without fragments) in water at the exact location of detector position (centre of the SOBP) in a volume with dimensions $1 \mathrm{~cm} \times 1 \mathrm{~cm}$ (perpendicular to the beam) $\times 0.1$ $\mathrm{cm}$ (in the beam direction). The fluence averaged LET ( $\mathrm{LET}_{\mathrm{f}}$ ) and dose averaged LET $\left(\mathrm{LET}_{\mathrm{d}}\right)$ were calculated for protons only (without fragments) and tabulated in table 2.

\section{Results}

\subsection{Dosimeter repeatability, reproducibility and batch reproducibility}

As shown in table 3 the dosimeter repeatability was lowest for alanine dosimeters from ISS (0.4\%) while it was $0.6 \%$ and $1.6 \%$ for $\mathrm{UH}$ and IFJ PAN, respectively. The batch reproducibility was lowest for $\mathrm{UH}$ $(0.5 \%)$ while it was $0.9 \%$ and $2.6 \%$ for ISS and IFJ PAN, respectively. The relatively higher uncertainties for IFJ PAN were induced by the instability of the spectrometer (3.5\%).

For RPL-GD, the repeatability (5 consecutive readouts of the same detector) was found to be very good $(0.2 \%)$, while the dosimeter and batch reproducibility were $2.4 \%$ and $3.2 \%$ respectively (see table 3 ).

For TLDs, we performed a dosimeter reproducibility test, by irradiating the same dosimeters 5 times and reading the signal, which resulted in a dosimeter reproducibility of $1.8 \%$ for MCP-N and $0.7 \%$ for MTS-N. The batch reproducibility was $1.9 \%$ and $1.4 \%$ for MCP-N and MTS-N

Table 4

Measured to reference dose ratio of alanine dosimeters (IFJ PAN, ISS and UH) in Co-60 in PSDL (TRS-398).

\begin{tabular}{llll}
\hline & Alanine/EPR & & \\
\cline { 2 - 4 } & IFJ PAN & ISS & UH \\
\hline Calibration & Proton & Co-60 & Co-60 \\
Measured to reference dose ratio & 1.008 & 1.003 & 1.005 \\
Total relative uncertainty (\%) & $2.2 \%$ & $0.5 \%$ & $0.4 \%$ \\
\hline
\end{tabular}

Table 3

Dosimeter repeatability, reproducibility and batch reproducibility for different dosimetry systems and different institutes involving specific protocols.

\begin{tabular}{|c|c|c|c|c|c|c|c|}
\hline & \multicolumn{3}{|l|}{ EPR } & \multirow{2}{*}{$\begin{array}{l}\text { RPL-GD } \\
\text { GD-352M }\end{array}$} & \multicolumn{2}{|l|}{ TLD } & \multirow{2}{*}{$\frac{\mathrm{OSL}}{\mathrm{Al}_{2} \mathrm{O}_{3}: \mathrm{C}}$} \\
\hline & Ala (IFJ PAN) & Ala (ISS) & Ala (UH) & & MCP-N & MTS-N & \\
\hline Dosimeter repeatability & $1.6 \%$ & $0.4 \%$ & $0.6 \%$ & $0.2 \%$ & & & \\
\hline Dosimeter reproducibility & & & & $2.4 \%$ & $1.8 \%$ & $2.4 \%$ & $4.9 \%$ \\
\hline Batch reproducibility & $2.6 \%$ & $0.9 \%$ & $0.5 \%$ & $3.2 \%$ & $1.9 \%$ & $1.4 \%$ & $4.4 \%$ \\
\hline
\end{tabular}


respectively. OSL resulted in the worst dosimeter reproducibility (4.9\%) and batch reproducibility (4.4\%).

\subsection{Dosimeter response in reference fields}

A comparison among alanine calibrations used by IFJ PAN, ISS and UH was performed at the Italian PSDL according to TRS-398 (see table 4). It showed a very good agreement to the reference Co-60 dose (10 Gy) within $0.8,0.3$ and $0.5 \%$ for IFJ PAN, ISS and UH, respectively. Between the institutes the agreement was excellent (coefficient of variation of $0.2 \%)$.

\subsection{Dosimeter response in pencil beam scanning}

\subsubsection{Uncertainties of dosimetry systems}

Uncertainty on the proton beam dose was $0.61 \%$ (coverage factor, $\mathrm{k}$ $=1$ ), as measured with ionization chamber for 3 mono-energetic proton beams. Uncertainty in detector positioning inside the PTW phantom is not specified by the manufacturer. However a paper comparing the uncertainty on the ionization chamber positioning in different types of phantoms reports a chamber positioning uncertainty of $0.3 \mathrm{~mm}$ for the PTW 41023 [32]. The specific holders used in our study may slightly alter this positioning uncertainty but it is not expected that this will be largely different than the positioning uncertainty of a chamber [32]. The alanine uncertainties reflect uncertainties on the dose estimation intrinsic to the institutes' protocols and calibration which was $1 \%$ for ISS, $0.45-0.7 \%$ for UH, $4.2 \%$ for IFJ PAN at $10 \mathrm{~Gy}$. For IFJ PAN the main source of uncertainty results from the instability of the spectrometer $(3.5 \%)$. The uncertainties shown in table 5 reflect these uncertainties as well as the uncertainty on the proton beam delivery $(0.6 \%)$. When comparing the uncertainties on the proton dose the IFJ PAN has largest uncertainties, which on average was $4.2 \%$, when compared to $1.2 \%$ for ISS and $0.8 \%$ for UH as calibrated in Co- 60 . The conversion of alanine data of ISS and UH from Co- 60 calibrated data to PT calibrated data increased the average uncertainties to $1.6 \%$ and $1.0 \%$ for ISS and UH proton calibrated data. The analysis of the uncertainty in dose calculations for RPL-GD is based on the scheme presented by Knežević at al. [28]. In this study the GD-352M average uncertainty was $1.9 \%$ including

Table 5

Ratio of Alanine measured doses to TPS dose for different beam parameters (varying ranges and modulations). Results for different institutes (IFJ PAN, ISS, $\mathrm{UH}$ ) and calibration methods Co-60 calibration (not for IFJ PAN) and proton calibration are shown, including underneath the total relative uncertainty on the dose calculations.

\begin{tabular}{|c|c|c|c|c|c|}
\hline Beam characteristics & $\begin{array}{l}\text { IFJ - } \\
\text { proton }\end{array}$ & $\begin{array}{l}\text { ISS - } \\
\text { Co-60 }\end{array}$ & $\begin{array}{l}\text { ISS - } \\
\text { Proton }\end{array}$ & $\begin{array}{l}\text { UH - } \\
\text { Co-60 }\end{array}$ & $\begin{array}{l}\text { UH - } \\
\text { Proton }\end{array}$ \\
\hline $\begin{array}{l}\text { Range and } \\
\underline{\text { Modulation }(\mathrm{cm})}\end{array}$ & \multicolumn{5}{|c|}{ Ratio between measured and TPS dose } \\
\hline R20M5 & 0.99 & 0.98 & 1.00 & 0.97 & 1.00 \\
\hline R20M10 & 0.99 & 0.99 & 1.00 & 0.98 & 1.01 \\
\hline R20M15 & 1.00 & 0.99 & 1.01 & 0.98 & 1.02 \\
\hline R20M20 & 1.00 & 0.99 & 1.00 & 0.99 & 1.02 \\
\hline R5M5 & 1.01 & 0.97 & 0.99 & 0.97 & 1.00 \\
\hline R10M5 & 1.00 & 0.98 & 0.99 & 0.97 & 1.01 \\
\hline R15M5 & 0.99 & 0.98 & 0.99 & 0.97 & 1.00 \\
\hline R20M5 & 0.99 & 0.98 & 0.99 & 0.97 & 1.01 \\
\hline R25M5 & 0.98 & 0.98 & 0.99 & 0.97 & 1.00 \\
\hline $\begin{array}{l}\text { Range and } \\
\text { Modulation }(\mathrm{cm})\end{array}$ & \multicolumn{5}{|c|}{ Total relative uncertainties on dose } \\
\hline R20M5 & $4.2 \%$ & $1.2 \%$ & $1.6 \%$ & $0.8 \%$ & $1.0 \%$ \\
\hline R20M10 & $4.2 \%$ & $1.2 \%$ & $1.6 \%$ & $0.8 \%$ & $1.0 \%$ \\
\hline R20M15 & $4.2 \%$ & $1.2 \%$ & $1.6 \%$ & $0.8 \%$ & $1.0 \%$ \\
\hline R20M20 & $4.2 \%$ & $1.2 \%$ & $1.6 \%$ & $0.8 \%$ & $1.0 \%$ \\
\hline R5M5 & $4.2 \%$ & $1.2 \%$ & $1.6 \%$ & $0.9 \%$ & $1.0 \%$ \\
\hline R10M5 & $4.2 \%$ & $1.2 \%$ & $1.6 \%$ & $0.8 \%$ & $1.0 \%$ \\
\hline R15M5 & $4.2 \%$ & $1.2 \%$ & $1.6 \%$ & $0.9 \%$ & $1.0 \%$ \\
\hline R20M5 & $4.2 \%$ & $1.2 \%$ & $1.6 \%$ & $0.9 \%$ & $1.0 \%$ \\
\hline R25M5 & $4.2 \%$ & $1.2 \%$ & $1.6 \%$ & $0.9 \%$ & $1.0 \%$ \\
\hline
\end{tabular}

the spread of 3 detectors (on average $0.9 \%$ ), the Co- 60 calibration $(1.3 \%)$ and proton dose $(0.61 \%)$ uncertainty. The mean total uncertainty of MCP-N and $\mathrm{Al}_{2} \mathrm{O}_{3}: \mathrm{C}$ detectors was $5.0 \%$ and $5.6 \%$ which includes the spread of the 6 different detectors on the average $(3.4 \%$ and $4.6 \%$, respectively) as well as the proton dose uncertainty $(0.61 \%)$ and calibration uncertainty ( $3.55 \%$ and $2.97 \%$, respectively). The calibration uncertainty included the Co-60 irradiation uncertainty (2\%) and spread on calibration detectors $(2.9 \%$ and $2.2 \%)$. For MTS-N the average uncertainty was $2.7 \%$, including the spread of 6 detectors $(1.6 \%)$, the proton dose uncertainty $(0.61 \%)$ and the calibration uncertainty $(2.1 \%)$.

\subsubsection{Alanine response in $P T$}

The agreement between measured and TPS dose was measured for alanine detectors following the protocols for proton calibration (IFJ PAN, ISS and UH) and Co-60 (ISS and UH), as shown in table 5. Following Co-60 calibration the measured doses in PT were up to $3.3 \%$ and $2.8 \%$ lower than TPS dose for UH and ISS respectively both in R5M5, corresponding to the configuration with the highest $\mathrm{LET}_{\mathrm{f}}$ values $(1.62 \mathrm{keV} / \mu \mathrm{m})$. On the other hand the configuration R20M20 with the lowest $\mathrm{LET}_{\mathrm{f}}$ values $(0.89 \mathrm{keV} / \mu \mathrm{m})$ demonstrated almost perfect agreement between the measured and TPS doses (within 0.01\%). On average, the difference compared to TPS dose was $1.8 \%$ for alanine pellets from ISS and $2.5 \%$ for alanine pellets from $\mathrm{UH}$ as calibrated with Co-60. Nevertheless when performing a proton calibration the agreement between measured and TPS dose was on average $0.8 \%, 0.7 \%$ and $0.8 \%$ with maximum difference of $-2.2 \%$ (R25M5), $-1.5 \%$ (R5M5) and $+2.2 \%$ (R20M20) for IFJ PAN, ISS and UH respectively.

\subsubsection{Luminescence dosimetry response in $P T$}

The RPL-GD, TLD (MCP-N and MTS-N) and OSLD results are shown in table 6 presenting the ratio of measured to TPS doses and the total relative uncertainties from the different detector systems. A mean under response of $18 \%$ was observed for GD-352M, with a minimal rise in the response for increased modulation from 0.81 for R20M5 to 0.86 for R20M20. An even larger under response of measured dose compared to TPS is observed for MCP-N, which ranged from $17 \%$ for R20M20 to $27 \%$ for R5M5. These MCP-N data confirm that the response is inversely proportional to the LET of the protons, as shown also by Sądel et al [33], and demonstrated in the next section through the dosimeter response as function of $\mathrm{LET}_{\mathrm{f}}$. The MTS-N measured a higher dose than TPS for SOBP

Table 6

Ratio of measured to TPS dose for RPL-GD, TL (MCP-N and MTS-N) and OSL detectors for different beam parameters (varying ranges and modulations), including (underneath) the total relative uncertainty on the dose calculations (Co-60 calibration).

\begin{tabular}{|c|c|c|c|c|}
\hline \multirow{3}{*}{$\begin{array}{l}\text { Beam characteristics } \\
\text { Range and Modulation }(\mathrm{cm})\end{array}$} & \multirow{2}{*}{$\begin{array}{l}\text { RPL } \\
\text { GD-352M }\end{array}$} & \multicolumn{2}{|l|}{ TL } & \multirow{2}{*}{$\begin{array}{l}\text { OSL } \\
\text { Luxel }\end{array}$} \\
\hline & & MCP-n & MTS-n & \\
\hline & \multicolumn{4}{|c|}{ Ratio between measured and TPS dose } \\
\hline R20M5 & 0.81 & 0.76 & 1.06 & 0.95 \\
\hline R20M10 & 0.84 & 0.79 & 1.02 & 0.93 \\
\hline R20M15 & 0.84 & 0.82 & 1.05 & 0.95 \\
\hline R20M20 & 0.86 & 0.83 & 1.04 & 0.97 \\
\hline R5M5 & 0.80 & 0.73 & 1.11 & 0.87 \\
\hline R10M5 & 0.80 & 0.75 & 1.07 & 0.97 \\
\hline R15M5 & 0.80 & 0.76 & 1.07 & 0.97 \\
\hline R20M5 & 0.81 & 0.76 & 1.06 & 0.93 \\
\hline R25M5 & 0.81 & 0.76 & 1.07 & 0.91 \\
\hline 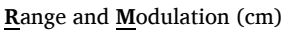 & \multicolumn{4}{|c|}{ Total relative uncertainties on dose } \\
\hline$\overline{\mathrm{R}} 20 \mathrm{M} 5$ & $1.6 \%$ & $4.5 \%$ & $2.5 \%$ & $5.4 \%$ \\
\hline R20M10 & $1.8 \%$ & $4.4 \%$ & $3.0 \%$ & $7.2 \%$ \\
\hline R20M15 & $1.7 \%$ & $5.5 \%$ & $2.3 \%$ & $4.8 \%$ \\
\hline R20M20 & $1.7 \%$ & $6.0 \%$ & $2.4 \%$ & $4.3 \%$ \\
\hline R5M5 & $1.9 \%$ & $5.0 \%$ & $4.0 \%$ & $6.2 \%$ \\
\hline R10M5 & $1.8 \%$ & $4.5 \%$ & $2.9 \%$ & $6.3 \%$ \\
\hline R15M5 & $2.5 \%$ & $5.9 \%$ & $2.9 \%$ & $3.6 \%$ \\
\hline R20M5 & $2.0 \%$ & $5.2 \%$ & $2.5 \%$ & $5.5 \%$ \\
\hline R25M5 & $2.3 \%$ & $4.0 \%$ & $2.3 \%$ & $7.0 \%$ \\
\hline
\end{tabular}




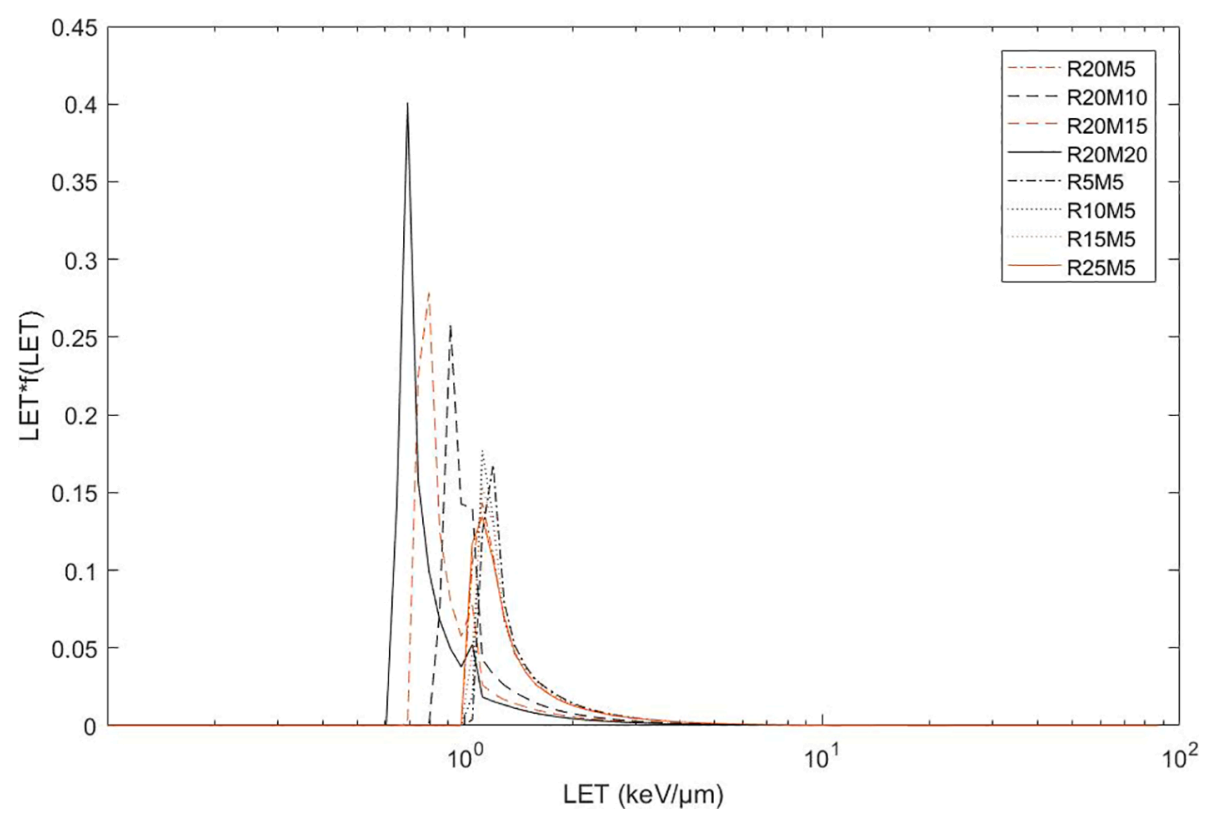

Fig. 2. The proton LET spectra for different configurations of range (R) and modulation (M) as simulated with Monte Carlo.

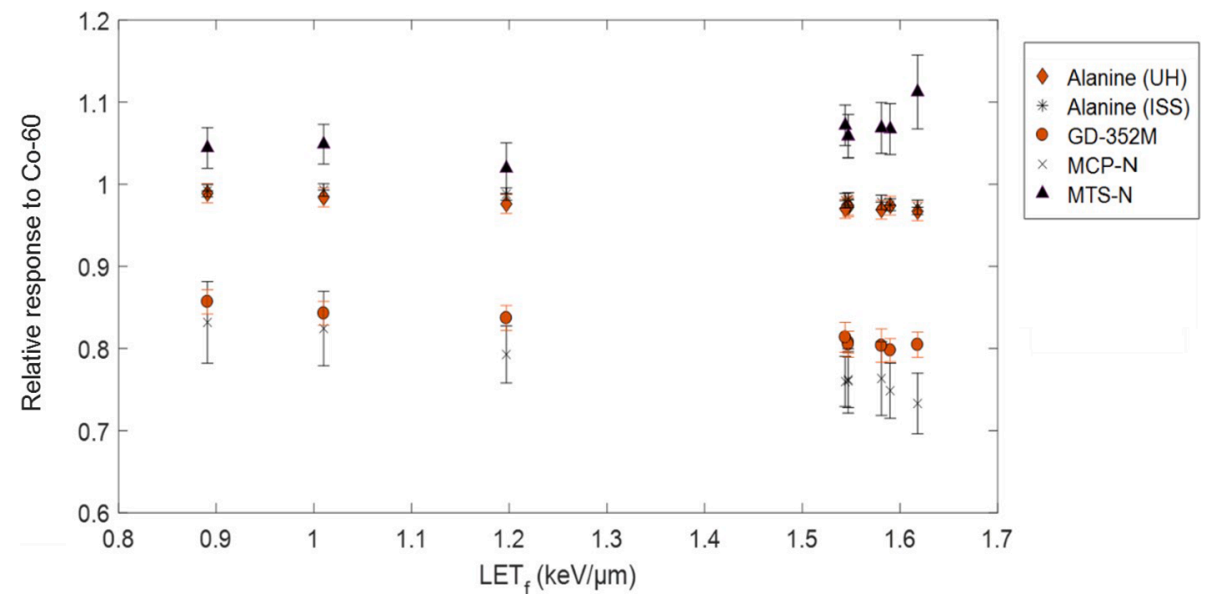

Fig. 3. Relative response compared to Co-60 as function of $\mathrm{LET}_{\mathrm{f}}$ for alanine detectors (UH and ISS), GD-352M, MCP-N and MTS-N dosimeters.

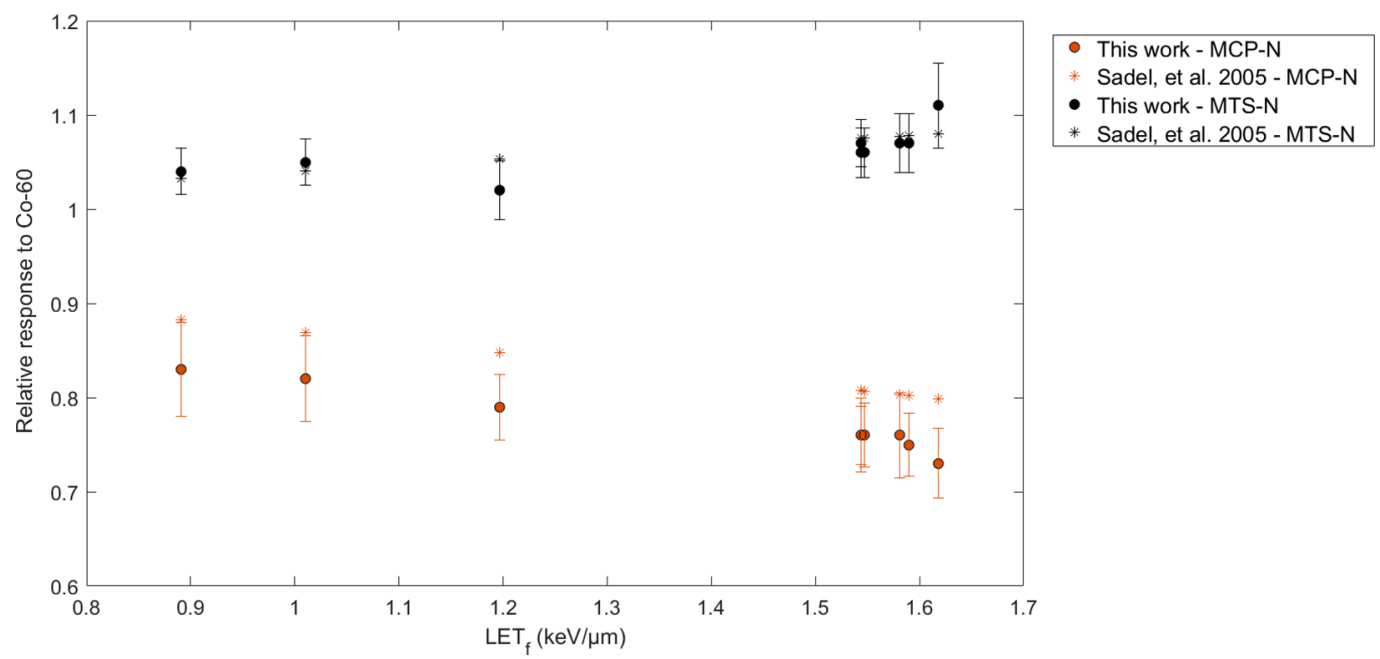

Fig. 4. Comparison of measured relative TL response to published data from Sądel, et al. [33]. For both MCP-N and MTS-N the experimental relative response data of this study as well as the published data from Sądel et al are plotted as function of $\mathrm{LET}_{\mathrm{f}}$. 
ranges and modulations corresponding to elevated $\mathrm{LET}_{\mathrm{f}}$ of protons. The highest MTS-N dose compared to TPS (11.2\%) was observed for R5M5, which corresponded to $\mathrm{LET}_{\mathrm{f}}$ of $1.62 \mathrm{keV} / \mu \mathrm{m}$. The OSLD dosimeters demonstrate a mean under response of $6.3 \%$ while no different response was observed when changing modulation or range, which can be due to the large uncertainties for this particular detector.

\subsubsection{Dosimeter response as function of $L E T_{f}$}

The proton fluence-averaged LET spectra in Fig. 2 show that increasing the modulation from 5 to $20 \mathrm{~cm}$ for a fixed range $(20 \mathrm{~cm})$ respectively decreased the $\mathrm{LET}_{\mathrm{f}}$ from $1.55 \mathrm{keV} / \mu \mathrm{m}$ to $0.89 \mathrm{keV} / \mu \mathrm{m}$ in the centre of the SOBP. On the other hand, a fixed modulation of $5 \mathrm{~cm}$ with varying ranges shows only a very slight decrease of the $\mathrm{LET}_{\mathrm{f}}$ in the centre of the SOBP, from $1.62 \mathrm{keV} / \mu \mathrm{m}$ for R5M5 to $1.54 \mathrm{keV} / \mu \mathrm{m}$ for R25M5. The dosimeter response, expressed as relative response compared to Co60, is shown in Fig. 3 for alanine detectors as calibrated in Co-60, and therefore is limited to UH and ISS. Also, GD-352M, MCP-N and MTS-N data are plotted as function of $\mathrm{LET}_{\mathrm{f}}$, suggesting an LET dependence.

The TL luminescence response data for MCP-N and MTS-N are also plotted, together with published data from the study of Sądel et al. 2005, in Fig. 4, demonstrating a similar change of the TL response as function of $\mathrm{LET}_{\mathrm{f}}$. Nevertheless this work shows MCP-N TL response data that were on average $6 \%$ lower than the published data [33]. On the other hand, the MTS-N showed very good agreement within $2 \%$.

\section{Discussion}

A program for mailed auditing of proton therapy is crucial for dosimetry harmonisation among proton therapy centres which is currently challenged by the lack of international and national primary dose standards for proton beams. Within EURADOS (WG9) different passive detector systems have been tested in pencil beam scanning to allow intercomparison of operating systems and procedures in use by different institutes as well as comparisons between different techniques such as EPR, TL, RPL and OSL.

\subsection{Alanine-EPR dosimetry systems of 3 European institutes}

To our knowledge, this is the first study that systematically compares alanine-EPR dosimetry systems of 3 European institutes and their response in pencil beam scanning, for potential implementation of a mailed dosimetry auditing system in PT. The participating institutes have a long-standing experience in alanine EPR dosimetry for pencil beam scanning (IFJ PAN, Poland) and also for national auditing programs in conventional radiotherapy (UHasselt and ISS) [4,5]. These institutes use slightly different types of alanine pellets manufactured by Gamma-service (IFJ PAN and ISS) and Harwell (UH) with slightly larger cylindrical shape, $3 \mathrm{~mm}$ versus $2.7 \mathrm{~mm}$ respectively, while the composition was $96 \%$ versus $90.9 \%$ of alanine and $4 \%$ versus $9.1 \%$ of ligand material/paraffin respectively. Slightly different protocols and practices are used as well as different EPR readers, which are described in detail in this manuscript. For IFJ PAN a calibration curve has been generated in the institute using the $60 \mathrm{MeV}$ proton beam due to the lack of a PSDL. In the case of UH and ISS the calibration curve has been generated from irradiation in a Co-60 source in a PSDL, namely PTB (Germany) and ENEA-INMRI (Italy) for UH and ISS respectively. Therefore, the traceability to the primary Co- 60 standards was different between UH and ISS results and the IFJ PAN results were traceable to the SSDL in Warsaw. Nevertheless, we performed irradiations in the Italian PSDL (ENEAINMRI) which revealed an excellent agreement between the institutes for Co-60 (within $0.21 \%$ ). Dosimeter repeatability of 10 consecutive readings was below $1 \%$ for ISS and UH while it was clearly higher for IFJ PAN (1.6\%). This can be explained by the spectrometer instability in IFJ PAN (3.5\%). Also the batch reproducibility reflected this instability for IFJ PAN (2.6\%) when compared to values below $1 \%$ for UH and ISS. In protons, alanine pellets showed a decreased response compared to Co-60 which was mostly pronounced for the configuration R5M5 (2.8 and $3.3 \%$ for ISS and UH respectively) corresponding to the highest $\mathrm{LET}_{\mathrm{f}}$ (1.62 keV/um). On the other hand, a better agreement $(<1.2 \%)$ was seen for R20M20 corresponding to the lowest $\mathrm{LET}_{\mathrm{f}}(0.89 \mathrm{keV} / \mathrm{um})$. This reflects a very limited dependence of the alanine response as function of LET. In the literature, a decreased relative response of the alanine dosimeters as function of LET has been described by Palmans et al. [34]. The relative response data ranged between 0.95 and 0.99 for proton energies from $24 \mathrm{MeV}$ to $60 \mathrm{MeV}$ [35-38], which corresponded to the levels of proton energies considered in our study for calibration $(26 \mathrm{MeV}$ average energy in the SOBP) and during pencil beam scanning (40-100 $\mathrm{MeV}$ ).

Following calibration in a proton beam the agreement between the measured doses for all alanine pellets and the TPS dose, was significantly improved and on average the difference was below $0.8 \%$. Nevertheless, for some configurations the deviation was above $2 \%$ for IFJ PAN reaching up to $-2.3 \%$ (R25M5) and for $\mathrm{UH}$ reaching up to $+2.2 \%$ (R20M20). This latter may be explained by the different $\mathrm{LET}_{\mathrm{d}}$ in water of the calibration beam $(2.93 \mathrm{keV} / \mu \mathrm{m})$ which was higher than the $\mathrm{LET}_{\mathrm{d}}$ calculated in the different pencil beam scanning configuration $(2.49-1.44 \mathrm{keV} / \mu \mathrm{m})$ and more specifically for R20M20 this corresponded to the lowest $\mathrm{LET}_{\mathrm{d}}(1.44 \mathrm{keV} / \mu \mathrm{m})$.

Not all alanine detectors were calibrated in Co- 60 because IFJ PAN only uses proton calibration detectors and at the time of the experiment no traceable Co- 60 source was available. Therefore the alanine detectors were all calibrated additionally in proton beams at a later time, requiring extra irradiations in IFJ PAN which allowed comparison of the response between alanine detectors.

Despite the high stability of the alanine pellets and the good performance in pencil beam scanning, some practical aspects need to be considered for mailed auditing such as the need for a high dose irradiation to allow accurate absolute dosimetry ( $\geq 10 \mathrm{~Gy}$ ) as well as the sensitivity of these detectors to temperature and humidity. Unlike EPR, luminescence detector systems are easier to use and more readily available. Even though the dose required is lower than for alanine detectors, the uncertainties associated to the readout process and batch inhomogeneities are generally higher than for alanine/EPR system.

\subsection{Luminescent based dosimetry systems}

Luminescence dosimetry systems such as RPL and OSL are emerging, while TLDs are widely available and are extensively used for auditing conventional radiotherapy centers. In this study we tested the response of MTS-N, MCP-N, GD-352M and $\mathrm{Al}_{2} \mathrm{O}_{3}$ :C detectors, for mail auditing in PT. Surprisingly, the current study shows that the spread in the detector readings for protons are higher for MCP-N (on average $3.4 \pm 1.0 \%$ ) compared to the data derived from Co-60 systematic analysis of batch reproducibility (1.9\%) with individual sensitivity correction (i.e. use of IF). The number of detectors used for the reproducibility analysis was 10 while we irradiated 6 detectors in the proton therapy experiment. Also, it must be noted that the use of IFs in protons may not be entirely correct because these are derived from Co-60 irradiations. Indeed the use of IF in proton therapy did not significantly reduce the mean spread (on average from $3.1 \pm 0.5 \%$ to $3.4 \pm 1.0 \%$ ) while it was significantly reduced (paired $t$-test $\mathrm{p}=0.01)$ in Co-60 irradiations $(2.6 \pm 0.35 \%$ to $1.9 \pm$ $0.3 \%)$. On the other hand, the sensitivity spread of TLD detectors during PT irradiations may be due to different positioning of detectors within the holder. Nevertheless, this will be very small, because the holder precisely fits the TLD detectors and a small shift in position will probably not give rise to large deviations because we measured in the centre of the SOBP and small position changes will only slightly alter the $\mathrm{LET}_{\mathrm{f}}$. The spread of the MTS-N detectors were also higher in PT irradiations (1.6\%) compared to Co-60 (0.7\%) which again may be related to the use of IF that may not fully apply for proton irradiations but it may also be related to positioning errors. Also beam non-uniformities during pencil beam scanning may lead to an increased spread of detector data. Nevertheless, 
this would mean it should be seen by all other detector systems, which was not observed. For OSLD a comparable detector spread in both protons and photons $( \pm 4.5 \%)$ was observed. This large uncertainty for OSLD is mainly due to the readout process which was performed in an in-house reading system and for which the laser, used to read the detectors, is not very stable. Additionally, the shape and dimensions of the in-house detectors are not completely identical. Unfortunately, this effect cannot be taken into account because no IFs are used for OSLDs. We consider that both effects, laser instability and no IFs used, cause the large spread in OSLD data. For the RPL GD-352M detectors the average spread of the detector data was lower (0.9\%) compared to the spread of 10 detectors irradiated in Co-60 (3.4\%). For the RPL-GD detectors IF was not used and should be taken into consideration for auditing purposes where high measurement precision is needed. The readout can also be influenced by the positioning and number of the glass dosimeters in the reading tray which can accommodate up to 20 dosimeters. The sensitive area of the glass is $6 \mathrm{~mm}$ and the alignment of the glass dosimeter readout area within the tray towards the laser in the reader will affect the reading as well. In the literature the position dependency of the magazine is observed, and in some cases because the magazine is made of plastic (resin) a small change of the shape of the magazine can occur and influence the readout area and values [17]. The deviation of the readout values due to loading position can be caused by the UV laser beam, photomultiplier tube and effective reading point [17]. The accuracy and high precision of the readout values depend on stable operation of the Dose Ace reader which could differ among different readers. These, and other factors, such as magazine correction factors and individual factors, should be taken into account for high dose precision measurements. It is recommended that the optimum reading method and corrections factors should be tested and set in order to minimize the uncertainties in dose readout values, to allow this system to be used for mail auditing in PT.

Our data demonstrate an underestimation of TPS dose for the GD$352 \mathrm{M}$, MCP-N and $\mathrm{Al}_{2} \mathrm{O}_{3}: \mathrm{C}$ detectors, due to a decreased detector response in PT compared to Co-60. The most pronounced under response was seen for MCP-N (up to 27\%) for which response largely depends on the particle LET as published in previous data from Sądel et al [33]. Nevertheless the response of MCP-N measured in our study was slightly lower (5-8\%) compared to the published data [33]. For example, for the same $\mathrm{LET}_{\mathrm{f}}$ of $1.5 \mathrm{keV} / \mu \mathrm{m}$ the relative response was 0.76 in our study compared to 0.81 in the study from Sacdel et al [33]. This can be due to the anomalous low temperature anomalous behavior of LiF: $\mathrm{Mg}, \mathrm{Cu}, \mathrm{P}$ detectors when exposed to light particles if a post-irradiation pre-readout protocol is not applied, or because response corrections for the material composing the detectors are not performed [39]. The MTS-N data in this work were in very good agreement with published data and demonstrated an increased response compared to TPS for SOBP configurations with elevated $\mathrm{LET}_{\mathrm{f}}$. The lower response of OSLD in PT compared to Co-60 was small, on average $6.3 \%$ and maximally $10 \%$ and did not show an LET dependence. In conclusion, both TL systems studied show LET dependence and will require the implementation of correction factors when used for mail auditing. In the literature a slight energy dependence is shown for $\mathrm{Al}_{2} \mathrm{O}_{3}: \mathrm{C}$ detectors which depended largely on the quantification parameter used [40]. Nevertheless response in proton beam was not studied in that work but was analyzed using a low-LET irradiation $\left({ }^{90} \mathrm{Sr}\right.$ beta rays) and heavy charged particle beams including $150 \mathrm{MeV} / \mathrm{u}{ }^{4} \mathrm{He}, 400 \mathrm{MeV} / \mathrm{u}{ }^{12} \mathrm{C}, 490 \mathrm{MeV} / \mathrm{u}^{28} \mathrm{Si}, 500 \mathrm{MeV} / \mathrm{u}$ ${ }^{56} \mathrm{Fe}$ covering a range of LET in water from $0.2 \mathrm{keV} / \mu \mathrm{m}$ to $198 \mathrm{keV} / \mu \mathrm{m}$. For this reason and due to the fact that the response is largely affected by the reader set-up (detector positioning, stimulation light, optical filtration, quantum response of the reader, etc.) it is difficult to compare our results directly to available literature data. The GD-352M detector demonstrated a decreased response in PT compared to Co-60 (on average $18 \%$ ) which seemed to be slightly dependent on the proton energy. The data in the literature on the characterization of glass dosimeters in proton beams are very limited. These data report results for another type of RPL dosimeter without energy compensation filter (GD301) for which the detector response is calibrated in proton beams in comparison to the current study where calibration is performed in Co-60 beams. The results from the literature demonstrated an energy dependence below $3 \%$ in comparisons to ionization chamber measurements $[41,42]$. In the phantom study measured doses with RPL-GD dosimeters type GD-301 at isocentre of SOBP were within 5\% in comparison to calculated doses [42]. Also these results showed that the GD-301 type is relatively independent on LET $[41,42]$. Our study used a GD-352M type of glass dosimeter with a plastic holder which contains a filter and the measured results showed a $5.8 \%$ decrease in the response when increasing the modulation and consequently decreasing the $\mathrm{LET}_{\mathrm{f}}$ from 1.55 to $0.89 \mathrm{keV} / \mu \mathrm{m}$. Therefore, GD-352M glass dosimeters will also require the use of correction factors for their implementation as a mail auditing dosimeter.

\subsection{Future plans}

In the framework of EURADOS WG9, the next step is to set-up an auditing program for pencil beam scanning proton therapy centers in Europe. We are currently planning a test experiment to verify a new 3D printed system for positioning detectors in an available water phantom that will be shipped together with several types detector systems (Alanine, RPL, TL powder) to a limited number of proton therapy centers in Europe.

\section{Conclusions}

The current study evaluated the response of alanine, MTS-N, MCP-N, GD-352M and $\mathrm{Al}_{2} \mathrm{O}_{3}$ : $\mathrm{C}$ dosimeters in the same controlled conditions for possible application in a mailed audits in proton therapy. The study was also unique as it studied and compared the response of alanine detectors from three different institutes (ISS, Italy; UH, Belgium and IFJ PAN, Poland). From the specific systems and protocols used the study showed that alanine detectors are characterized by low uncertainties and weak energy dependence for different proton beams delivered with PBS, suggesting this detector as a good candidate for mailed auditing of proton therapy centres. However, luminescence detector systems are emerging and becoming more readily available. For these systems, more extensive calibrations and corrections are needed in order to be used for mailed dosimetry auditing in PT.

\section{References}

[1] Izewska J, Bokulic T, Kazantsev P, Wesolowska P, van der Merwe D. 50 Years of the IAEA/WHO postal dose audit programme for radiotherapy: what can we learn from 13756 results? Acta Oncol 2020;59(5):495-502.

[2] IAEA, Comprehensive Audits of Radiotherapy Practices: A Tool for Quality Improvement. 2007, Vienna: International Atomic Energy Agency.

[3] Ferreira IH, Dutreix A, Bridier A, Chavaudra J, Svensson H. The ESTRO-QUALity assurance network (EQUAL). Radiother Oncol 2000;55(3):273-84.

[4] De Angelis C, De Coste V, Fattibene P, Onori S, Petetti E. Use of alanine for dosimetry intercomparisons among Italian radiotherapy centers. Appl Radiat Isot 2005;62(2):261-5.

[5] Schaeken B, Cuypers R, Lelie S, Schroeyers W, Schreurs S, Janssens H, et al. Implementation of alanine/EPR as transfer dosimetry system in a radiotherapy audit programme in Belgium. Radiother Oncol 2011;99(1):94-6.

[6] Yegingil Z, DeWerd LA, Davis SD, Hammer C, Kunugi K. Photon beam audits for radiation therapy clinics: a pilot mailed dosemeter study in Turkey. Radiat Prot Dosim 2012;148:249-57.

[7] Homnick J, Ibbott G, Springer A and Aguirre J, THD352-05: Optically Stimulated Luminescence (OSL) Dosimeters Can Be Used for Remote Dosimetry Services. Vol. 35. 2008.

[8] TRS-398. Absorbed Dose Determination in External Beam Radiotherapy. International Atomic Energy Agency, Vienna 2000.

[9] Wesolowska PE, Cole A, Santos T, Bokulic T, Kazantsev P, Izewska J. Characterization of three solid state dosimetry systems for use in high energy photon dosimetry audits in radiotherapy. Rad Meas 2017;106:556-62.

[10] Izewska J, Andreo P. The IAEA/WHO TLD postal programme for radiotherapy hospitals. Radiother Oncol 2000;54(1):65-72.

[11] Mehta K, Girzikowsky R. Alanine-ESR dosimetry for radiotherapy IAEA experience. Appl Radiat Isot 1996;47(11-12):1189-91. 
[12] Bulski W, Chełmiński K, Rostkowska J. Dosimetry audit of radiotherapy treatment planning systems. Radiat Prot Dosim 2015;165(1-4):472-6.

[13] Slusarczyk-kacprzyk W, Bulski W, Ulkowski P, Chelminski K. [P162] Postal TLD dosimetry audits in radiotherapy in poland - Results of 2017. Phys Med 2018;52: 145. https://doi.org/10.1016/j.ejmp.2018.06.463.

[14] Yukihara EG, McKeever SWS. Optically stimulated luminescence (OSL) dosimetry in medicine. Phys Med Biol 2008;53(20):R351-79.

[15] Ahmed MF, Shrestha N, Schnell E, Ahmad S, Akselrod MS, Yukihara EG. Characterization of $\mathrm{Al}(2) \mathrm{O}(3)$ optically stimulated luminescence films for $2 \mathrm{D}$ dosimetry using a 6 MV photon beam. Phys Med Biol 2016;61(21):7551-70.

[16] Araki F, Moribe N, Shimonobou T, Yamashita Y. Dosimetric properties of radiophospholuminescent glass rod detector in high-energy photon beams from a linear accelarator and Cyber-Knife. Med Phys 2004;31:1980-6.

[17] Son K, Jung H, Shin SH, Lee H-H. Evaluation of the dosimetric characteristics of a radiophotoluminescence glass dosimeter for high-energy photon and electron beams in the field of radiotherapy. Rad Meas 2011;46:1117-22.

[18] Rah J-E, Kim S, Cheong K-H, Lee J-W, Chung J-B, Shin D-O, et al. Feasibility study of radiophotoluminescent glass rod dosimeter postal dose intercomparison for high energy photon beam. Appl Radiat Isot 2009;67(2):324-8.

[19] Mizuno H, Kanai T, Kusano Y, Ko S, Ono M, Fukumura A, et al. Feasibility study of glass dosimeter postal dosimetry audit of high-energy radiotherapy photon beams. Radiother Oncol 2008;86(2):258-63.

[20] Izewska J, Bera P, Vatnitsky S. IAEA/WHO TLD postal dose audit service and high precision measurements for radiotherapy level dosimetry. International Atomic Energy Agency/World Health Organization. Radiat Prot Dosim 2002;101(1): 387-92.

[21] De Angelis C, Fattibene P, Onori S, Petetti E, Bartolotta A, Santamaria AS. Transferability of ASTM/NIST alanine-polyethylene recipe at ISS. Appl Radiat Isot 2000;52(5):1197-201.

[22] Nagy V. Accuracy considerations in EPR dosimetry. Appl Radiat Isot 2000;52(5): 1039-50.

[23] Anton M. Development of a secondary standard for the absorbed dose to water based on the alanine EPR dosimetry system. Appl Radiat Isot 2005;62(5):779-95.

[24] Glass AT. Glass dosemeter GD-300 series-Instruction Manual. 2012.

[25] Vekic B, Ban R, Miljanic S. Secondary Standard Dosimetry Laboratory at the Ruder Boskovic Institute, Zagreb. Arh Hig Rada Toksikol 2006;57:189-94.

[26] Miljanic S. ntercomparison of dosimeters for non-targetorgan dose measurements in radiotherapy-activity of EURADOS WG9: Radiation protection in medicine. Proceedings of The 6th Inter-national Workshop on Individual Monitoring of Ionizing Radiation, Oarai, Japan 2010; 95-107.

[27] NIST. Tables of X-ray mass attenuation coefficients and mass-energy absorption coefficients. Natl Inst Stand Technol 2008.
[28] Knežević Ž, Stolarczyk L, Bessieres I, Bordy JM, Miljanić S, Olko P. Photon dosimetry methods outside the target volume in radiation therapy: Optically stimulated luminescence (OSL), thermoluminescence (TL) and radiophotoluminescence (RPL) dosimetry. Rad Meas 2013;57:9-18.

[29] Yukihara EG, McKeever SWS. Optically stimulated luminescence: fundamentals and applications. Wiley; 2011. p. 378.

[30] Boons R. External and environmental radiation dosimetry with optically stimulated luminescent detection device developed at the SCK.CEN. J Nucl Sci Technol 2012; 2:6-15.

[31] Pelowitz DB, MCNPX USER'S MANUAL: Version 2.7.0, in Los Alamos National Laboratory report LA-CP-11-00438. 2011.

[32] Kinoshita N, Oguchi H, Adachi T, Shioura H, Kimura H. Uncertainty in positioning ion chamber at reference depth for various water phantoms. Rep Pract Oncol Radiother 2018;23(3):199-206.

[33] Sądel M, Bilski P, Swakoń J, Rydygier M, Horwacik T, Weber A. Comparative investigations of the relative thermoluminescent efficiency of LiF detectors to protons at different proton therapy facilities. Rad Meas 2015;82:8-13.

[34] Palmans H. Effect of alanine energy response and phantom material on depth dose measurements in ocular proton beams. Technol Cancer Res Treat 2003;2(6): 579-86.

[35] Mierzwińska G, Michalec B, Ogłodek I, Petelenz B, Waligórski MPR. Alanine/EPR dosimetry as a potential tool for quality assurance in proton beam radiotherapy. Rom Rep Phys 2014;66:54-60.

[36] Onori S, d'Errico F, De Angelis C, Egger E, Fattibene P, Janovsky I. Alanine dosimetry of proton therapy beams. Med Phys 1997;24(3):447-53.

[37] Cuttone G, Raffaele L, Tonghi L, Rovelli A, Sabini MG, Egger E, et al. First dosimetry intercomparison results for the CATANA project. Phys Med 1999;15: 121-30.

[38] Bartolotta A, Barone Tonghi L, Brai M, Cuttone G, Fattibene P, Onori S, et al. Response characteristics of thermoluminescence and alanine-based dosemeters to 16 and $25 \mathrm{MeV}$ proton beams. Radiat Prot Dosim 1999;85(1):353-6.

[39] Parisi A, de Freitas Nascimento L, Van Hoey O, Mégret P, Kitamura H, Kodaira S, et al. Low temperature thermoluminescence anomaly of $\mathrm{LiF}: \mathrm{Mg}, \mathrm{Cu}, \mathrm{P}$ radiation detectors exposed to $1 \mathrm{H}$ and $4 \mathrm{He}$ ions. Rad Meas 2018;119:155-65.

[40] Yukihara EG, Gaza R, McKeever SWS, Soares CG. Optically stimulated luminescence and thermoluminescence efficiencies for high-energy heavy charged particle irradiation in Al2O3:C. Rad Meas 2004;38(1):59-70.

[41] Rah J-E, Oh DH, Shin D, Kim D-H, Ji YH, Kim JW, et al. Dosimetric evaluation of a glass dosimeter for proton beam measurements. Appl Radiat Isot 2012;70(8): $1616-23$.

[42] Rah J-E, Oh DH, Shin D, Lee SB, Kim TH, Kim J-Y, et al. Feasibility study of glass dosimeter for postal dose intercomparison of high-energy proton therapy beams. Rad Meas 2013;59:66-72. 